\title{
STUDYING THE PROBABILITY OF ACQUIRING HIV IN A CERTAIN POPULATION
}

\author{
Rafael Mateus Carrión \\ Mathematical Engineering \\ Universidad EAFIT \\ rmateusc@eafit.edu.co
}

\author{
Daniel Otero Gómez \\ Mathematical Engineering \\ Universidad EAFIT \\ doterog@eafit.edu.co
}

\author{
Henry Laniado \\ Sciences Department \\ Universidad EAFIT \\ hlaniado@eafit.edu.co
}

\section{Contents:}

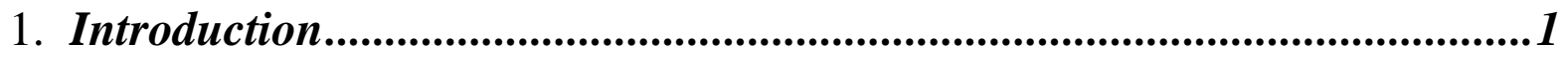

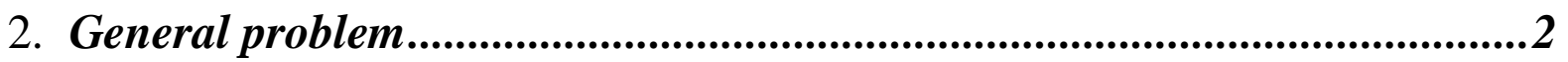

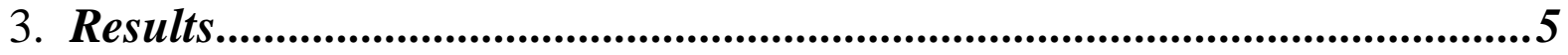

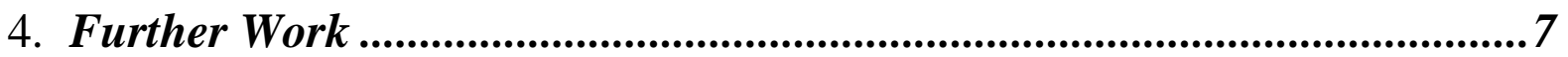

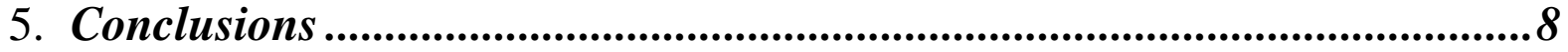

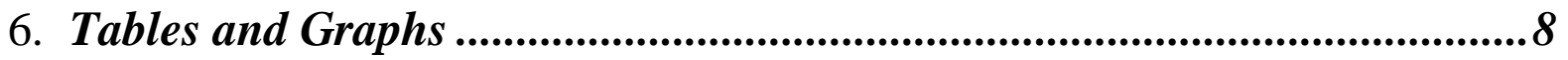

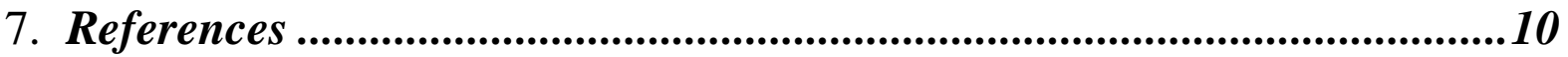

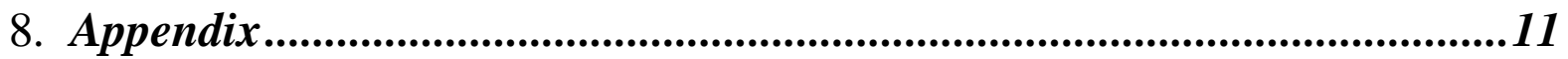

\begin{abstract}
This document is aimed at the study of the probability of acquiring the HIV virus at a certain population. A meticulous analysis is included regarding the understanding had, in order to design an accurate probabilistic model for each scenario. Based on a data recollection regarding HIV transmission, various specific situations were proposed to study deeper how vulnerable is an individual of acquiring HIV according to the number of interactions and the type of encounter had with an HIV carrier.
\end{abstract}

\section{Introduction}

Since the early 1980s, the world has disputed an exhausting fight against the prevailing pandemic of HIV/AIDS. It has reached millions of people across the globe, leaving 77.3 million people infected with HIV, and near of 35.4 million deaths due to AIDS-related illness [1]. Undoubtedly, this virus has had a global impact on public health, and as a result, it has raised a worldwide concern regarding the well-being of the population.

The human immunodeficiency virus (HIV) is a virus that attacks the CD4 cells (or T cells), who are in charge of fighting diseases and infections, affecting the immune system. The development of the virus has three phases, being AIDS the last and the severest of all. During this phase, the 
immune system has already been heavily damaged, turning the body extremely vulnerable to opportunistic illnesses [2].

The transmission of this pathogen occurs when there is interaction between infected body fluids. The chances of acquiring this virus depend on the encounter had with the HIV carrier. Risk factors as direct blood contact and avoidance of barrier methods such as condom usage increase these chances [3].

\section{General problem}

In order to make it easier for the readers to fully comprehend how the model was built up, we will start presenting a series of simple models thought for very specific situations that increase in complexity as the papers goes on. The purpose of doing such thing relies on the expectation that the work exposed in this paper can be easily read by a large group of people and encourage them to create and adapt similar models that may be useful for similar problems regarding absorbent states.

Every complex problem can be solved if it is first reduced into much simpler situations that allow to gain a greater understanding of the topic. The first situation that we will take into account is the following: A susceptible person has a sexual partner that is infected with HIV and they have $n$ sexual encounters with the same behavior. This is easy to represent by defining a succession of events $\left\{E_{n}\right\}, n \in \mathbb{N}$. In which every event $E$ can take the form of $F$ (Failure), that represent a sexual encounter in which HIV was not acquired, and the form of $S$ (Success), which stands for a sexual encounter in which HIV was acquired.

$\begin{array}{cccccc}E_{1} & E_{2} & E_{3} & E_{4} & \cdots & E_{n} \\ F_{1} & F_{2} & F_{3} & F_{4} & \cdots & F_{n} \\ S_{1} & N_{1} & N_{2} & N_{3} & \cdots & N_{n-1} \\ F_{1} & S_{1} & N_{1} & N_{2} & \cdots & N_{n-2} \\ F_{1} & F_{2} & S_{1} & N_{1} & \cdots & N_{n-3} \\ \vdots & \vdots & \vdots & \vdots & \ddots & \vdots \\ F_{1} & F_{2} & F_{3} & F_{4} & \cdots & S_{1}\end{array}$

Figure 1. Matrix of possible results of the succession $\left\{E_{n}\right\}$, defined above

As we can appreciate in Figure 1, there exist only two possible outcomes: acquiring HIV or not. The letter $\mathrm{N}$ in the figure represent that both sexual partners are infected with the virus, so the probability of getting it is 1 . N stands for "null", since the virus has been already transmitted, the probability of acquiring the disease is no longer relevant. The simplest problem that we can solve concerning this situation is to find out what is the probability of getting infected. This is a simple model, which structure will be similar to the Bernoulli distribution, as it only contemplates two results of a certain behavior.

Firstly, we would have to assign a certain probability of contracting the disease or not after a sexual encounter. It is important to clarify that the probability of transmitting the virus through sexual intercourse is not always the same. This depends on the role that a person is playing during the 
encounter, insertive or receptive, whether the intercourse is anal, oral or vaginal, the risk factors that the person is exposing to, like the use of a condom or not, and the stage of HIV in which the infected partner currently is.

Being stated that the probability of getting the virus varies depending on several factors, we can embrace entirely every possibility by defining it as a letter. Thus, let us define $p$ as the probability of not acquiring the virus after $\mathrm{n}$ sexual encounters, and (1-p) the probability of acquiring it. If we establish $n=1$, the probability function will look like Equation 1, shown above.

$$
f(x)=\left\{\begin{aligned}
p, & x=0 \\
1-p, & x=1 \\
0, & \text { otherwise }
\end{aligned}\right.
$$

Equation 1. Probability function for each encounter with a person suffering from HIV.

Again, this would be the simplest situation that we would ever have, the way of representing is extremely obvious. However, if we do not assign a specific value to $n$, the problem will not be much harder to represent through a function. It is not hard to see that the probability of not acquiring the virus after $n$ encounters is simply $p^{n}$. Now, the moment in which we consider the possibility of getting the virus is when it gets tricky. There are exactly $\mathrm{n}$ ways of getting the virus. If we look to Figure 1, we can appreciate that the sexual encounter in which the virus was transmitted could be any of the encounters that the person had. So, the probability of acquiring the virus is the summation of the probability of acquiring HIV in each of the $\mathrm{n}$ encounters. The probability function for $n$ encounters will be the following:

$$
f(x)=\left\{\begin{aligned}
p^{n}, & x=0 \\
\sum_{i=1}^{n}(1-p) p^{n-i}, & x=1 \\
0, & \text { otherwise }
\end{aligned}\right.
$$

Equation 2. Probability function for $\boldsymbol{n}$ encounters with a person suffering from HIV, with the same probability each encounter

Note: Each of this functions are proved to be probability functions. Such proves can be found in the appendix.

Note: Notice that the procedure established to determine the probability of acquiring the virus can be seen as the summation of geometric probability functions that measure the number of sexual encounters until the virus is contracted.

Consider the situation of having n promiscuous sexual encounters with the same behavior. Namely, having a single sexual encounter with $\mathrm{n}$ different infected individuals which are in the same stage of HIV, having a unique type of intercourse, playing the same role, and exposing to the exact same risk factors. This situation is represented exactly the same as it is made in Figure 1, and its probability function would be Equation 2 as well.

Equation 2 could only be possible if each encounter has the sexual behavior with people in the same stage of HIV, therefore, the same probability. It is still possible to think in an even more real 
situation: having $\mathrm{n}$ promiscuous sexual encounters with infected people in different HIV stages and having different sexual behaviors. The probability at each encounter with an infected person will vary, since all the interaction will not always be the same. The probability function of the model if the probability vary with each encounter will be as follows:

$$
f(x)= \begin{cases}\prod_{i=0}^{n} p_{i}, & x=0 \\ \sum_{i=1}^{n}\left(1-p_{i}\right) \prod_{j=0}^{i-1} p_{j}, & \left.x=1 \quad \text { (with } p_{0}=1\right) \\ 0, & \text { otherwise }\end{cases}
$$

Equation 3. Probability function for $\boldsymbol{n}$ encounters with a person suffering from HIV, with a variation in the probability each interaction.

Note: This model requires to know the exact probability of transmission in each sexual encounter. Hence, it is necessary to know what behavior had every sexual encounter. A model that does not need this information will be of great difficulty to build, so it would not be considered in this paper.

Before going on it is of great relevance to talk about the trait of absorbency that this type of problem has, which is one of the most important characteristics of HIV. The nature of the virus makes that once you are infected with the disease you can never stop having it, as you cannot go back from the stage of HIV you have developed yet. For instance, once you develop AIDS, which correspond to stage three of HIV, you can never go back to stage two. The quality of not being able to go back to a previous state once you got a specific one is called absorbency. This is clearly evidenced in Figure 1, yet it can be represented in a simpler way using the following Markov Model, that represent the change between states.

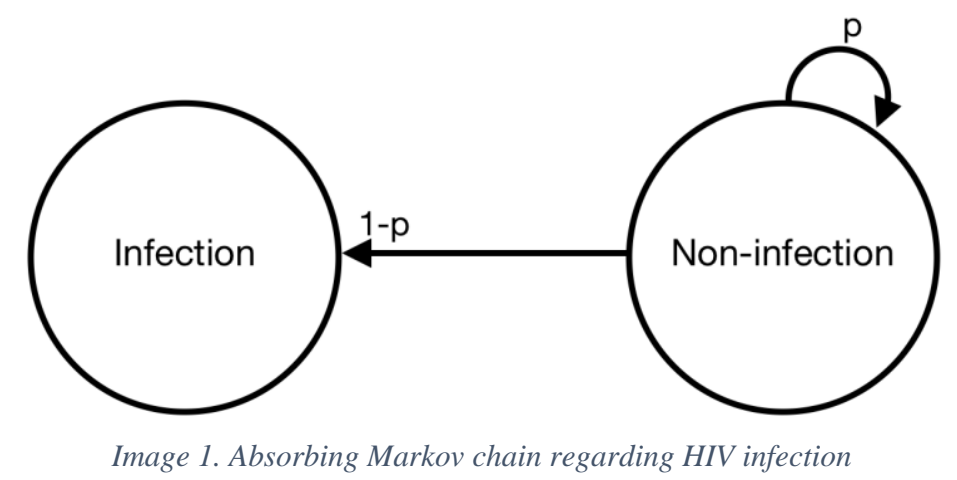

As it can be seen in Image 1 the Markov chain in this model, can be identified as an absorbing model, as once an individual person reaches the state of infection it stays permanently at the corresponding state. Likewise, the particular individual can be at the state on non-infection unlimited times, therefore, the transition matrix of this problem will be as shown in Image 2 . 


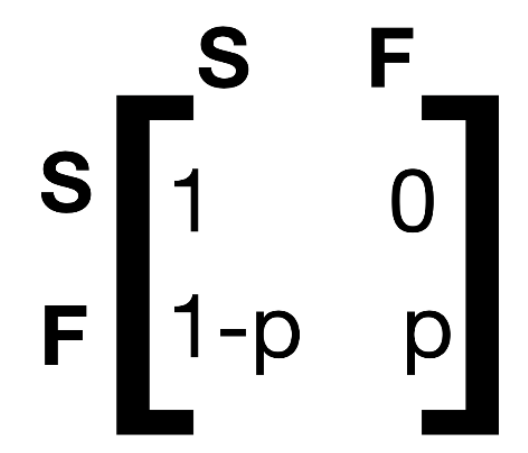

Image 2.Transition matrix of the corresponding Markov model

The transition matrix of this model, following the notation used before, consider $S$ as the successful event (HIV infection) and $F$ as the failure event (non-HIV infection). As explained before, once an individual gets infected its probability of getting infected will be 1, its probability of noninfection will be 0 , due to the fact that once infected, an individual can never go to the state of noninfection again. Then, its probability of getting infected depends of $p$, which is the probability of getting infected in each interaction, thus, the probability on non-infection will be its compliment $(1-\mathrm{p})$.

\section{Results}

For a population of size $A$, with $B$ people infected with HIV virus and $A$ - $B$ susceptible population (vulnerable people to acquire the virus), to calculate the probability of getting infected with HIV depends on various factors. For better understanding, we will see it as a chain of events as follows:

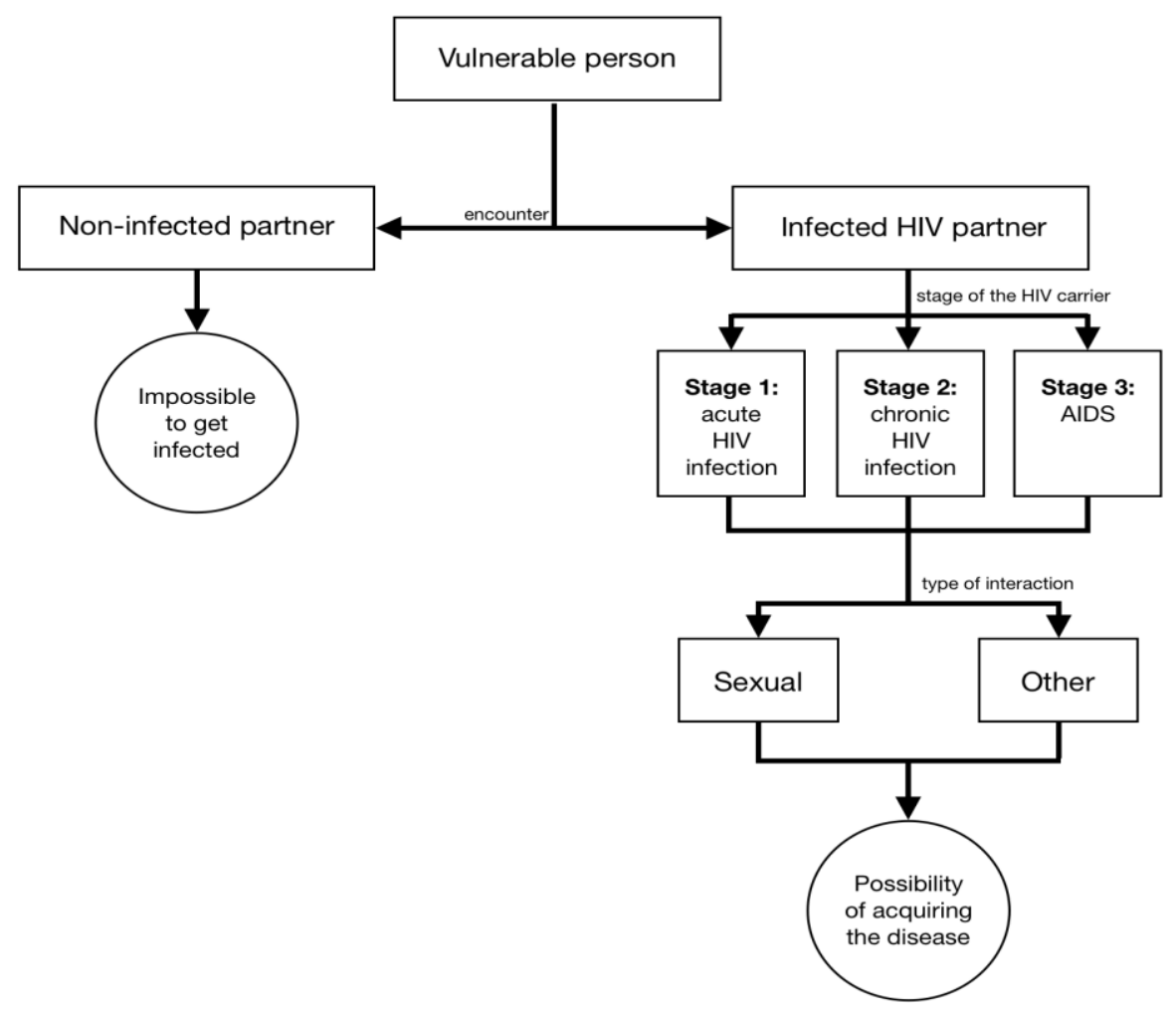

Image 3. Illustration of the vulnerability of an individual person to become infected with HIV. 
Firstly, there is the possibility that the susceptible individual has no interaction with an infected person, then, there is no chance that this specific individual becomes infected with this virus. On the other hand, if this person interacts with an HIV carrier, there exists the possibility of getting infected. The probability of the encounter is:

$$
P(X=x)=\frac{B}{A-1}, A>0
$$

Equation 3. Probability of having an encounter with an HIV positive person in a population A with B infected.

This possibility depends on the stage of the disease its partner is, and what type of interaction has with it [4], we consider other interaction as: blood transfusion, needle-sharing during injection and infected needle-stick usage (This paper would not take into account parent-to-child transmissions). As can be seen in Table 1, the probability varies according to the type of encounter had with the infected person.

For simplicity reasons, external factors that affect the propagation of this disease are not considered, since it can increase, significantly, the complexity of this model. As an example, if we consider $\mathrm{ART}^{1}$ in this problem, the chances that an infected HIV person transmits the disease, reduce considerably. Therefore, after an interaction with an HIV positive person, we would have to analyze if such individual does this treatment to predict the chances of infection [5].

Now if we consider that our population to study is an entirely men population, and the encounters they have are always sexually related. There will be fewer restrictions on our model, as there will be only one way of spreading the disease (insertive or receptive anal intercourse). If we suppose that a person had $n$ sexual encounters in which he was the passive partner ${ }^{2}$, then, the probability of spreading the disease will be 0.0138 (see Table 1). Then, based on Equation 2, the probability function corresponding to the estimation of the probability of getting infected of HIV in $n$ sexual encounters will be as follows:

$$
f(x)=\left\{\begin{aligned}
0.9862^{n}, & x=0 \\
1-0.9862^{n}, & x=1 \\
0, & \text { otherwise }
\end{aligned}\right.
$$

Equation 4. Probability function

Then, if we suppose that an individual has 50 receptive sexual encounters, the probability of acquiring or not the disease is shown in Graph 1. As can be evidenced, the probability of HIV acquisition increase with each encounter, this makes perfect sense, since with each encounter, the individual will increase its vulnerability. According to the probability of the interaction its graph will vary respectively.

Now, if we take into account the usage of protective barriers when having a promiscuous activity, such as condoms, then the probability of acquiring the disease in the same scenario as the above,

\footnotetext{
${ }^{1}$ Antiretroviral therapy (ART) is a medicine treatment use to treat HIV [5].

2 Passive partner $=$ receptive partner
} 
the probability will reduce an average of $65 \%^{3}$ [6]. As can be evidenced in Graph 2, having the same 50 interactions, the curves of acquisition and not acquisition change drastically as the probability, having the same interaction, reduces to 0.01104 .

\section{Further Work}

Going back to the situation presented before, where it is contemplated the probability of encounter with an HIV infected person. Building a function around this factor will give permit us establish our model around a more realistic situation. Having $\mathrm{n}$ promiscuous sexual encounters with the same behavior. Such situation was considered before in this paper without acknowledging the possibility of having a sexual encounter with a non-infected person. In order to do so we will have to redefine the concept of $\mathrm{F}$ and $\mathrm{S}$ as it is shown above, and add the probability of encounter with an infected person to the concepts:

$$
R=\frac{\text { Infeceted Population }}{\text { Total Population }}
$$

Image 4. Probability of encounter with an infected person, defined with $R$
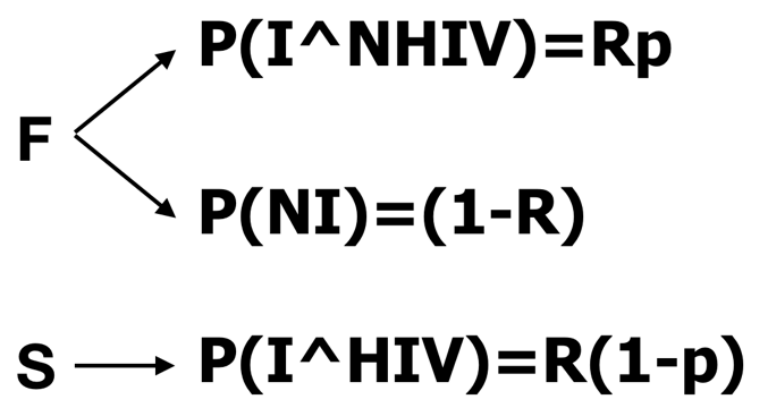

Image 5. Illustration of the probability of HIV acquisition in a population.

As it is clearly exposed in Image 5, a failed encounter can represent having sexual intercourse with an infected person and not getting infected or having sexual intercourse with a non-infected person. The probability of having successful encounter changed as well, representing having a sexual encounter with an infected person and acquiring the virus. In order to keep the model simple $\mathrm{R}$ is defined without subtracting the person which is having the encounters and, as it has the encounters, the number of people with which he/she had sex. This implies that we assume that the number of sexual encounters represent an insignificant fraction of the total population. $\mathrm{R}$ remains constant.

Now that we have established that there are two ways of having a failed encounter, the easier way of redefining the probability function is to reanalyze the way of not acquiring HIV can go. The

\footnotetext{
${ }^{3}$ If used correctly, condoms could reduce the chances of getting HIV from 90 to $95 \%$. But as not all people use them correctly, the effectiveness will not be as high as the previous, in fact it reduces to 60 to $70 \%$ [6]. We will be using $65 \%$ for easier calculation.
} 
succession of events will go the same as it is shown in Figure 1. On the row of uniquely failed there can be $z$ sexual encounters with infected people and $n-z$ encounters with non-infected people. The probability of not contracting the virus is them the summation of every possible value that $z$ can take in the interval $[0, n]$ as it is defined in Equation 5 above:

$$
\begin{gathered}
f(x)\left\{\begin{array}{c}
\sum_{z=0}^{n}\left(\begin{array}{c}
n \\
z
\end{array}\right)(r p)^{z}(1-r)^{n-z}, \quad x=0 \\
\sum_{y=1}^{n} r(1-p) \sum_{z=0}^{y-1}\left(\begin{array}{c}
y-1 \\
z
\end{array}\right)(r p)^{z}(1-r)^{y-1-z}, \quad x=1 \\
0, \quad \text { otherwise }
\end{array}\right. \\
\text { Equation 5. Probability function with probability of encounter included }
\end{gathered}
$$

Now that we have determined the way of defining a chain of failed encounters it is easy to find the function that determines the probability of contracting the virus. As it was stated before, the probability of acquiring the virus is the summation of the probabilities of being infected in each of the encounters, in other words, the summation of geometric probability functions. Analogously, our function will have the same structure, including the change of the way of defining the chain of failed encounters.

\section{Conclusions}

- In order to gain a greater understanding of diseases who possess the characteristic of absorbency, such as HIV, it is great importance to consider the simplest situation in which an individual can be infected, and start modifying the structure and assumptions as the situations are replaced for more complex ones. This would evidence a linearity between the models, which would be useful as a validation and guide when developing new ones.

- When considering the probability of being infected, disease who possess the characteristic of absorbency, such as other STD's, would always behave as a summation of geometric probability functions.

- Barrier usage, such as condom, is crucial for reducing the chances of getting infected with HIV. This can be clearly seen in the graphs shown in the Tables and Graphs section.

- It is evidenced that it is necessary to have a large number of risky sexual encounters to have a high probability of contracting the virus as well. Being promiscuous is the main risk factor that can lead to contracting the disease.

- The use of a condom slightly reduces the probability of getting the virus. At 50 sexual encounters it reduces the probabilities in less than a $10 \%$.

- The best way of preventing being infected with the disease is knowing with whom a person is having a sexual encounter due to the fact that this permits the person to have a basic a knowledge of what are the sexual behaviors of the other person, and determine if it is probable to acquire the virus. 


\section{Tables and Graphs}

\begin{tabular}{|c|c|c|c|c|}
\hline & Type of exposure & $\begin{array}{c}\text { Risk per } 10000 \\
\text { exposures }\end{array}$ & $\begin{array}{l}\text { 95\% confidence } \\
\text { interval }\end{array}$ & $\begin{array}{l}\text { Probability } \\
\text { of infection }\end{array}$ \\
\hline \multirow{5}{*}{ 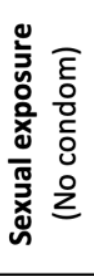 } & Receptive Anal Intercourse & 138 & $(102-186)$ & 0.0138 \\
\hline & Insertive Anal Intercourse & 11 & $(4-28)$ & 0.0011 \\
\hline & Receptive Penile-Vaginal Intercourse & 8 & $(6-11)$ & 0.0008 \\
\hline & Insertive Penile-Vaginal Intercourse & 4 & $(1-14)$ & 0.0004 \\
\hline & $\begin{array}{l}\text { Receptive Oral Intercourse } \\
\text { Insertive Oral Intercourse }\end{array}$ & \multicolumn{3}{|c|}{$\begin{array}{l}\text { Even though is very low, the posibility exists (of } \\
19000 \text { unprotected cases, not one positive) }\end{array}$} \\
\hline \multirow{3}{*}{ 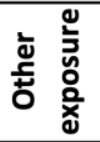 } & Blood Transfusion & 9250 & $(8900-9610)$ & 0.925 \\
\hline & Needle-sharing during injection use & 63 & $(41-92)$ & 0.0063 \\
\hline & Percutaneous(Needle-stick) & 23 & $(0-46)$ & 0.0023 \\
\hline
\end{tabular}

Table 1. Probabilities according to the interaction with an HIV positive person [3].

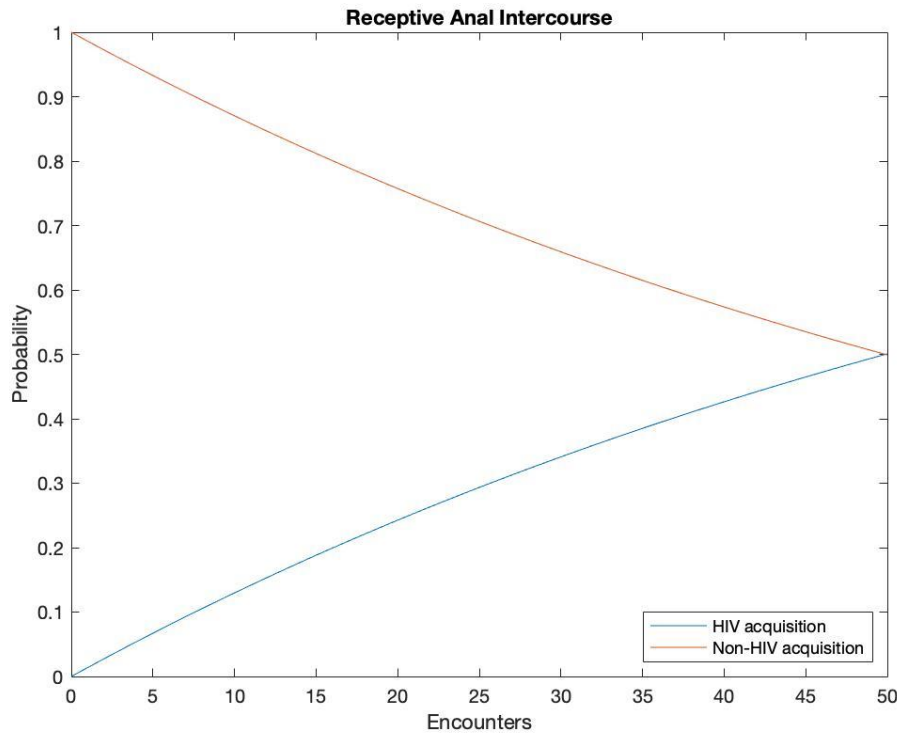

Graph 1. Probability graph regarding receptive anal intercourse in 50 interactions. 


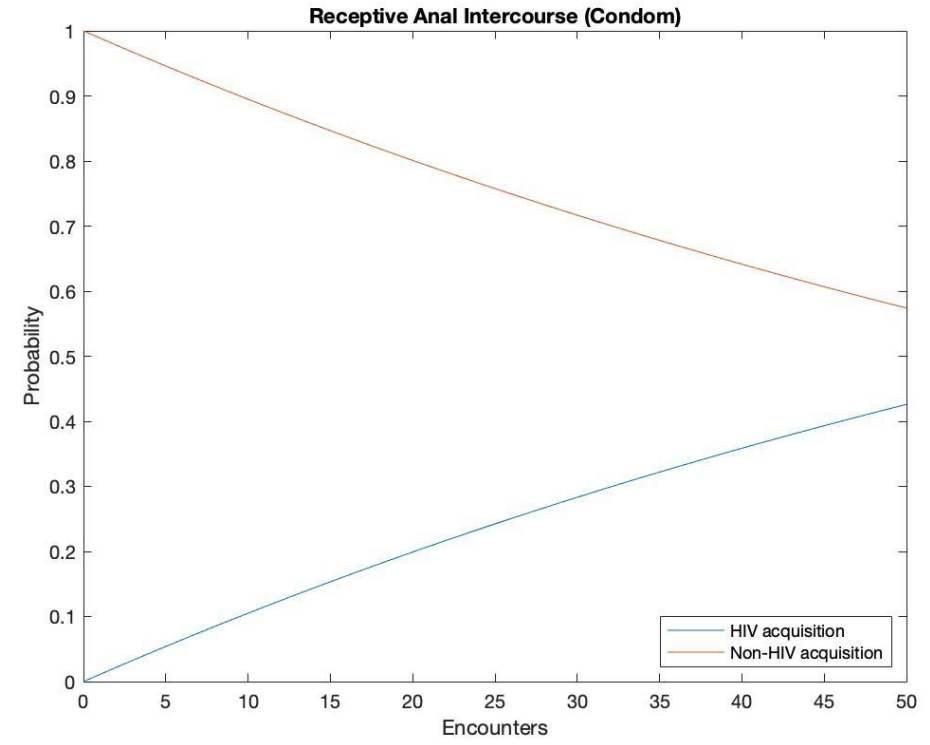

Graph 2. Probability graph regarding receptive anal intercourse in 50 interactions using condom.

\section{References}

[1] Avert. Global HIV and AIDS Statistics, 2018. https://www.avert.org/global-hiv-and-aidsstatistics, visited: 1/11/2019

[2] Center for Disease Control and Prevention. About HIV/AIDS, 2019. https://www.cdc.gov/hiv/basics/whatishiv.html, visited: 1/11/2019

[3] National Center for Biotechnology Information. Estimating per-act HIV transmission risk: a systematic review, 2014. https://www.ncbi.nlm.nih.gov/pmc/articles/PMC6195215/, visited: $1 / 11 / 2019$.

[4] National Center for Biotechnology. HIV Transmission by Stage of Infection and Pattern of Sexual Partnerships, 2010. https://www.ncbi.nlm.nih.gov/pmc/articles/PMC3018805/, visited: $1 / 11 / 19$

[5] Minority HIV/AIDS Fund. Understanding HIV Test Results, 2018. https://www.hiv.gov/hiv-basics/hiv-testing/learn-about-hiv-testing/understanding-hiv-testresults, visited: 4/11/2019.

[6] National Center for Biotechnology. Effectiveness of condoms in preventing HIV transmission, 1997. https://www.ncbi.nlm.nih.gov/pubmed/9141163 visited: 5/11/19 


\section{Appendix}

Proof Equation 2 sums 1:

$$
\begin{aligned}
p^{n}+\sum_{i=1}^{n}(1-p) p^{n-1} & = & p^{n}+(1-p) \sum_{i=1}^{n} p^{n-1} \\
& = & p^{n}+(1-p) \frac{1-p^{n}}{1-p} \\
& = & p^{n}+1-p^{n} \\
& = & 1
\end{aligned}
$$

Proof Equation 3 sums 1:

$$
\begin{aligned}
p^{n}+\sum_{i=1}^{n}(1-p) p^{n-1} & =\prod_{i=1}^{n} p_{i}+\sum_{i=1}^{n}\left(1-p_{i}\right) \prod_{j=0}^{i-1} p_{j} \\
& =\prod_{i=1}^{n} p_{i}+\left[\left(1-p_{1}\right) p_{0}+\left(1-p_{2}\right) p_{1} p_{0}+\cdots+\left(1-p_{n}\right) \prod_{i=0}^{n-1} p_{i}\right] \\
& =\prod_{i=1}^{n} p_{i}+\left[p_{0}-p_{1} p_{0}+p_{1} p_{0}-p_{2} p_{1} p_{0}+\cdots+\prod_{i=0}^{n-1} p_{i}-\prod_{i=1}^{n} p_{i}\right] \\
& =\prod_{i=1}^{n} p_{i}+\left[\begin{array}{c}
\left.p_{0}-\prod_{i=1}^{n} p_{i}\right] \\
p_{0} \\
1
\end{array}\right. \\
& =
\end{aligned}
$$

Proof Equation 5 sums 1:

$$
\sum_{z=0}^{n}\left(\begin{array}{l}
n \\
z
\end{array}\right)(r p)^{z}(1-r)^{n-z}+\left(1-\sum_{z=0}^{n}\left(\begin{array}{l}
n \\
z
\end{array}\right)(r p)^{z}(1-r)^{n-z}\right)=1
$$

